\title{
Educação permanente em saúde: experiência dos profissionais do serviço de atendimento móvel de urgência
}

Recebido em: 21/02/2013

Aprovado em: 25/11/2014
Glória Maria Pinto Coelho' Simone de Campos Vieira Abib ${ }^{2}$

Kátia Simoni Bezerra Lima ${ }^{3}$ Rodrigo Nonato Coelho Mendes ${ }^{4}$ Rafaela Ayanne Alves dos Santos ${ }^{5}$

Adriana Gonçalves de Barros ${ }^{6}$

Resumo: O estudo teve como objetivo descrever a experiência da Educação Permanente em Saúde vivenciada pelos profissionais de saúde de um serviço pré-hospitalar móvel de urgência. Estudo descritivo, de natureza qualitativa, desenvolvido com 36 profissionais do SAMU-192 de Juazeiro-BA. Os dados foram obtidos mediante entrevista semi-estruturada, e analisados por meio da análise de conteúdo. O núcleo de educação do serviço não se encontra implantado e a sobrecarga de trabalho foi apontada como um desafio para a consolidação. Conclui-se que estratégias educativas são componentes essenciais para o fortalecimento e melhoria do componente pré hospitalar móvel de atenção às urgências.

Descritores: Educação em Saúde, Socorro de Urgência, Educação Continuada

\section{Standing in health education: experience of health professionals of pre-hospital care service}

Abstract: The study aimed to analyze the experience of Continuing Education in Health experienced by health care professionals in assisting the pre-hospital mobile emergency in the municipality of Juazeiro-BA. Descriptive and Quantitative research, developed with 36 professional working in the SAMU-192 to Juazeiro-BA. Data were obtained through interviews using a semi-structured, between September and October 2010, and analyzed using content analysis. For most respondents, the term Continuing Education in Health has an ongoing process of meaning within the required service in terms of individual growth and not as a process of collective change. Conclude that the found a profile of professionals interested and willing to achieving the Education Center in the SAMU emergency Juazeiro-BA, which is not deployed.

Descriptors: Health Education, Emergency Relief, Education Continuing

\section{Educación permanente para la salud: experiencia de los profesionales de atención móvil de emergencia}

Resumen: Analizar la experiencia de Educación Continua en Salud experimentado por profesionales de la salud en la asistencia de emergencia pre-hospitalaria móvil en el municipio de Juazeiro-BA. Métodos: La investigación cualitativa, de tipo exploratorio y descriptivo, desarrollado con 36 profesionales que trabajan en el SAMU-192 a Juazeiro-BA. Los datos se obtuvieron a través de entrevistas semi-estructurada, entre septiembre y octubre de 2010, y se analizaron mediante análisis de contenido. Resultados: Para la mayoría de los encuestados, el término Educación Continua en Salud cuenta con un proceso continuo de significado dentro del servicio requerido en términos de crecimiento individual y no como un proceso de cambio colectivo. Conclusión: Se encontró un perfil de los profesionales interesados y dispuestos a lograr el Centro de Educación en la emergencia SAMU Juazeiro-BA, que no se ha implementado.

Descriptores: Educación en Salud, Socorro de Urgencia, Educación Continua

\section{INTRODUÇÃO}

$\mathrm{E}$ m 1988, foi promulgada a nova Constituição Federal do Brasil. Entre suas determinações, estabeleceram-se as bases para a edição da Lei 8080/90, que instituiu o Sistema Único de Saúde (SUS). A Legislação do SUS, embora não tenha discorrido especificamente sobre a atenção às urgências, definiu as competências de cada esfera de governo, delegando aos gestores federais, estaduais e municipais, e aos respectivos Conselhos de Saúde a autonomia para a edição e aplicação de medidas, normas técnicas e estratégias visando garantir as diretrizes de universalidade, igualdade de acesso e integralidade da assistência ${ }^{(1)}$.

Apesar disso, elevados índices de óbitos e sequelas incapacitantes decorrentes dos agravos de saúde, bem como a superlotação de setores isolados da rede de atenção às urgências demandaram uma reorganização e aperfeiçoamento dos serviços que compõem essa rede. Com esta intenção, foi instituída a Política Nacional de Atenção às Urgências (PNAU). Também, toda e qualquer melhoria decorrente do uso de educação precede subsídios teóricos para tal.
E para fortalecer essas iniciativas, foi publicada a Portaria 2048/2002 emanada do Ministério da Saúde (MS), que traz orientações sobre a Educação Permanente nos serviços ${ }^{(2)}$.

Essa questão educativa já é discutida desde quando a Organização Panamericana de Saúde (OPAS) trouxe, na década de 70, a idéia da Educação Permanente em Saúde segundo a proposta de Educação Permanente do Pessoal da Saúde, discutindo a presença do ensino-aprendizagem a partir de situações concretas, tornando-se um mecanismo mobilizador dos atores envolvidos no intuito de aumentar a qualidade dos serviços prestados ${ }^{(2-4)}$.

A pesquisa, teve como objetivo descrever a experiência da Educação Permanente em Saúde vivenciada pelos profissionais de saúde atuantes na assistência de um serviço de atendimento pré-hospitalar móvel de urgência.

\section{MÉTODOS}

Pesquisa qualitativa, exploratória e descritiva. O lócus do estudo foi o Serviço de Atendimento Móvel de Urgência (SAMU) do município

'Enfermeira. Mestre em Ciências da Saúde pela Universidade Federal de São Paulo - UNIFESP. Docente do Curso de Enfermagem da Universidade Federal do Vale do São Francisco - UNIVASF. Email: gloriapintocoelho@gmail.com;

${ }^{2}$ Médica. Doutora em Cirurgia Pediátrica pela Universidade Federal de São Paulo - UNIFESP.

${ }^{3}$ Enfermeira. Mestre em Ciências da Saúde. Docente do Curso de Enfermagem da Universidade Federal do Vale do São Francisco - UNIVASF

${ }^{4}$ Enfermeiro graduado pela UNIVASF. Residente em Gerência de Serviços de Enfermagem pela Universidade Estadual de Londrina - UEL.

${ }^{5}$ Enfermeira. Residente em Saúde da Mulher pelo Instituto de Medicina Integral Professor Fernando Figueira - IMIP;

${ }^{6}$ Enfermeira. Mestranda em Enfermagem pela Universidade Federal do Rio Grande do Norte - UFRN. 
de Juazeiro-BA. A amostra foi constituída por 36 profissionais de saúde (médicos, enfermeiros e técnicos de enfermagem) trabalhadores, implicando $72 \%$ da população total em estudo. Foram excluídos os profissionais que estavam de férias ou licenças durante o período e aqueles que não consentiram em participar da pesquisa.

Para a concretização do estudo houve anuência institucional, e foi aprovado pelo Comitê de Ética em Pesquisa Hospital São Paulo da Escola Paulista de Medicina sob o parecer $n^{\circ} 0585 / 10^{(5)}$.

A coleta de dados foi realizada no período de setembro e outubro do ano de 2010, utilizando-se de uma entrevista semi-estruturada, orientada por um roteiro ${ }^{(6)}$. $\mathrm{O}$ anonimato dos participantes foi mantido pela adoção de nomes fictícios, iniciados pela vogal $A$, vez que nenhum dos participantes responde pela denominação atribuída às falas, sendo respeitadas as questões de sexo. Para melhor compreensão dos dados qualitativos, optou-se por realizar a análise dos resultados com base na Análise de Conteúdo.

\section{RESULTADOS}

Após a leitura flutuante e categorização dos dados, tomando por base a Análise do Conteúdo, foram descritas as três categorias a saber: Educação permanente em saúde na visão dos profissionais de Atendimento Pré Hospitalar (APH); Mudanças percebidas no atendimento de urgência. E quatro subcategorias: Processo contínuo de aprendizagem; Capacitações dentro do contexto e realidade vivenciada pelo serviço.

\section{Educação Permanente em Saúde na Visão dos Profissionais de APH}

É importante investigar primeiramente a percepção dos profissionais em relação ao processo educativo no serviço.

\section{Processo contínuo de aprendizagem}

O termo Educação Permanente em Saúde para a maioria dos profissionais de saúde entrevistados do SAMU - Juazeiro/BA têm um significado de processo contínuo necessário dentro do serviço, como oportunidade de adquirir mais conhecimentos, e assim, crescer profissionalmente. Destaque para o conhecimento individual e não como um processo de mudança coletivo.

"Processo contínuo, necessário para qualificação do profissional no seu dia-a-dia". (Alan).

"É muito interessante, oportunidade de crescer profissionalmente, e só temos a ganhar, tanto nós profissionais quanto a comunidade". (Américo).

"Tem que ter educação para qualificar os profissionais, respeitando a área de atuação de cada um". (Alberta).

Houve relatos dos entrevistados que demonstraram a preocupação de se ter a Educação Permanente inserida no serviço justamente por ser, no seu entendimento, a área de Urgência e Emergência uma especialidade que se encontra em constante atualização.

"A saúde é uma evolução contínua que predispõe a necessidade de atualizar as práticas, eu trabalho há um bom tempo com traumatologia, e percebo a importância de estar aprimorando as técnicas para assim melhorar o atendimento no dia-a-dia". (Apolônio).

"A importância de estar sempre atualizando os profissionais com novos conhecimentos, uma vez que o suporte de atendimento estar sempre em constante mudança para melhor atendimento para assegurar a vida dos pacientes". (Alcides).

Os profissionais apontam à importância do processo de educação permanente para a melhoria das práticas em saúde. Este é um dado animador, uma vez que percebem e valorizam a educação permanente como importante ferramenta de trabalho.

\section{Mudanças Percebidas no Atendimento de Urgência}

Como uma das metas para a consolidação do SUS é a qualidade na assistência à saúde e a humanização dos serviços, um dos mecanismos bastante discutido e eficaz é a Educação Permanente em Saúde, por ser um processo educativo aplicado ao trabalho onde estabelece uma reflexão crítica entre os sujeitos, garantindo mudanças nas relações, nos processos, nos atos de saúde e nas pessoas, e uma melhor articulação dentro e fora das instituições ${ }^{(\mathbf{8})}$.

Foi encontrada nessa categoria que os cursos já realizados e aqueles em andamento, para a maioria dos entrevistados, vêm refletindo em mudanças percebidas nas atividades diárias individuais.

"Com certeza vem mudando sim, é percebida uma melhor atuação dos profissionais na prática."(Adelaide).

"O curso ainda está no $3^{\circ}$ módulo, mas teoricamente falando já esclareceu bastante." (Anelisa).

"Sim já começou a modificar, eu percebo no dia-a-dia ações que me fazem lembrar das aulas que eu tive." (Alba).

Relatos demonstram as diversas formas de atuação da educação presente no serviço, como motivação, interesse e aperfeiçoamento das práticas.

"Vem modificando, motivando o profissional, modificou o comportamento percebido pelas perguntas, pelo interesse em buscar conhecimento e na melhoria da condução dos veículos". (Alan).

"[...] às vezes temos vícios nas práticas que acabam sendo quebradas e assim modificando o nosso atendimento. Começamos a observar novas formas de atuar nas práticas que nos trazem até bem-estar e com isso melhora $100 \%$ o nosso atendimento". (Alcione).

\section{DISCUSSÃO}

No tocante a Educação Permanente em Serviços de Urgência e Emergência, por meio do Núcleo de Educação em Urgência (NEU) e o Núcleo de Educação Permanente (NEP) percebe-se que a transformação pessoal, profissional e social tão almejada se dá em todas as relações do sujeito, por meio da interação e aquisição de novos conhecimentos, atitudes e conceitos ${ }^{(10)}$. Para tal, esse compromisso pessoal deve ser aprendido e conquistado dentro do serviço, pois não é fácil mudar concepções e práticas antigas.

O enfoque da Educação Permanente apresenta-se de forma clara e objetiva, como uma forma de incorporar o ensino e o aprendizado à vida cotidiana do serviço pré-hospitalar no contexto real em que ocorrem, a partir da problematização do próprio fazer, onde os profissionais passam de receptores a construtores do conhecimento e de alternativas de ação ${ }^{(11)}$.

Contudo, percebe-se, ainda, o pensamento fragmentado, marcado pelo distanciamento das categorias profissionais e suas funções correspondentes. Esta situação faz com que não exista muitas vezes a interação interdisciplinar necessária para se criarem novas alternativas e programá-las ${ }^{(11)}$. Nesse ínterim, sabe-se que o ponto de partida da Educação Permanente em Saúde para direcionar o aprendizado e assim a transformação da realidade, é a problematização coletiva do cotidiano do trabalho em equipe na saúde ${ }^{(12)}$.

No intuito de aumentar a qualidade dos serviços prestados, a Educação Permanente em Saúde funciona como dispositivo de aproximação entre o cotidiano do profissional do SUS e as necessidades da população(12). Sendo incentivada a participação de todos os atores envolvidos em um processo de relações que deve ocorrer no interior das equipes em atuação conjunta inserida em 
uma determinada instituição, bem como nas práticas intersetoriais correspondente às políticas em que se inscrevem os atos de saúde, constituindo, então, o Sistema Único de Saúde em uma verdadeira rede-escola ${ }^{(4)}$.

Sabe-se que no processo educativo permanente vivenciado pelos profissionais de saúde é notória a percepção de mudanças de atitudes e de conceitos. Contudo, é necessário ressaltar a importância do compromisso pessoal a ser aprendido e a ser conquistado por esses profissionais; para isso faz-se necessário constituir no ambiente de trabalho a motivação, levando os profissionais de saúde a buscarem constantemente o desenvolvimento pessoal, profissional e social, enfim, o interesse de continuar a aprender ${ }^{(10,13)}$

Se o primeiro passo, é motivar o profissional, o segundo passo é justamente aproximar a educação com a vida cotidiana, levantar as reais necessidades da organização do serviço e das práticas rotineiras, prevendo transformar as situações diárias em aprendizagem ${ }^{(11)}$. Como caminho para solidificar tais aspectos, torna-se relevante a educação permanente dos profissionais de saúde, pois permite a qualificação da assistência prestada, o que integra a satisfação mútua dos sujeitos envolvidos nesse processo de cuidar(1).

Tratando-se de APH móvel, os treinamentos, as capacitações e as trocas de experiências devem passar por um processo de reflexão e de auto-análise, cuja ação promove a problematização da realidade vivenciada e que, por sua vez, necessitará ser trabalhada e transformada por toda a equipe que integra o serviço ${ }^{(4)}$. Uma das contribuições que a educação trás para dentro do serviço, é sem dúvida, a oportunidade do aprimoramento das competências e habilidades frente às diversas situações operativas e existenciais que demandam conhecimento prévio e atualizado ${ }^{(14)}$.

\section{CONSIDERAÇÕES FINAIS}

Foram encontrados alguns fatores que dificultam o processo educativo, apesar de os funcionários estarem interessados em promover o conhecimento no serviço. Entre os obstáculos, encontram-se vínculos empregatícios instáveis e baixa remuneração salarial para determinadas categorias profissionais, favorecendo a sobrecarga de trabalho, a desmotivação, e mesmo a falta de tempo para se dedicar aos estudos e aos grupos de debates.

Em relação aos treinamentos realizados, todos os entrevistados possuíam alguma capacitação referente ao APH na sua maioria ofertados por outras instituições pelas quais possuem vínculo empregatício, portanto percebe-se a necessidade de treinamentos e atualizações de forma contínua serem inseridos no serviço para que seus profissionais vivenciem de fato a educação permanente.

O que deve ser compreendido é que, tanto o NEU como a Educação Permanente em Saúde são mecanismos recentes, que precisam ser discutidos, trabalhados, construídos por toda a equipe que integra o serviço pré-hospitalar móvel. Por isso, a princípio deve-se surgir a vontade e a determinação de todos em investir em algo novo, desde o seu planejando, execução, até a sua avaliação, na espera constante de contribuições significativas na melhoria da qualidade de um serviço tão importante na rede de saúde.

\section{Referências}

1. Brasil. Ministério da Saúde. Lei no $8080 / 90$, de 19 de setembro de 1990. Dispõe sobre as condições para a promoção, proteção e recuperação da saúde, a organização e o funcionamento dos serviços correspondentes e dá outras providências. Ministério da Saúde [Internet]; 1990 [Cited 2010 July 9]. Available from: http:// www.planalto.gov.br/ccivil_03/leis/L8080.htm;

2. Brasil. Ministério da Saúde. Política Nacional de Atenção às Urgências. $3^{a}$ ed. ampl. Ministério da Saúde [Internet]; 2006 [Cited 2010 July 9]. Available from: http://portal.saude.gov.br/portal/ arquivos/pdf/Politica\%20Nacional.pdf;

3. Tronchin DMR, Kurcgant P. Gerenciamento em enfermagem. Rio de Janeiro: Guanabara Koogan; 2005.

4. Ceccim RB. Educação Permanente em Saúde: desafio ambicioso e necessário. Interface comun saúde educ. 2005 [Cited 2010 July 9]; 9 (16): 161-77. Available from: http://www.scielo.br/scielo. php?script=sci_pdf\&pid=S1414-32832005000100013\&lng=en\&nr $\mathrm{m}=\mathrm{iso} \&$ tlng=pt;

5. Brasil. Ministério da Saúde. Conselho Nacional de Saúde, Resolução 196/96. Diretrizes e Normas Regulamentadoras de Pesquisas envolvendo Seres Humanos. Conselho Nacional de Saúde [Internet]. 1996 [Cited 2010 July 9]. Available from: http:// conselho.saude.gov.br/resolucoes/reso_96.htm;

6. Minayo MCS. O desafio do conhecimento: pesquisa qualitativa em saúde. 10. Ed. São Paulo: Hucitec; 2007.

7. Kmeteuk OF. Pesquisa e Análise Estatística. Rio de Janeiro: Fundo de Cultura; 2005.

8. Carotta F, Kawamura D, Salazar J. Educação Permanente em Saúde: uma estratégia de gestão para pensar, refletir e construir práticas educativas e processos de trabalhos. Saúde e Sociedade.
2009 [Cited 2010 July 9]; 18 (1): 48-51. Available from: http://www. scielo.br/pdf/sausoc/v18s1/08.pdf.

9. Brasil. Ministério da Saúde. Secretaria de Gestão do Trabalho e da Educação na Saúde. Departamento de Gestão da Educação em Saúde. Ministério da Saúde; 2009. 65p. Available from: http://portal.saude. gov.br/portal/arquivos/pdf/volume9.pdf.

10. Paschoal AS, Mantovani MF, Méier MJ. Percepção da educação permanente, continuada e em serviço para enfermeiros de um

hospital de ensino. Rev Esc Enferm USP [Internet]. 2007 [Cited 2010 July 9], 41(3): 478-84. Available from: http://www.scielo.br/pdf/reeusp/ v41n3/19.pdf.

11. Jesus MCP, Figueiredo MAG, Santos SMR, Amaral AMM do, Rocha LO, Thiollent MJM. Educação permanente em enfermagem em um hospital universitário. Rev esc enferm USP [Internet]. 2011 [Cited 2012 Nov 7], 45(5): 1229-36. Available from: http://www.scielo.br/pdf/ reeusp/v45n5/v45n5a28.pdf.

12. Lopes SRS, Piovesan ETA, Melo LO, Pereira MF. Potencialidades da educação permanente para a transformação das práticas de saúde. Com ciências saúde. 2007 [cited 2010 July 09]; 18(2): 147-155. Available from: http://www.fepecs.edu.br/revista/Vol18_2art06.pdf.

13. Oliveira MAN. Educação à Distância como estratégia para educação permanente: possibilidades e desafios. Rev Bras Enferm. [Internet] 2007 [cited 2010 Jul 09]; 60(5): 585-9. Available from: http:// www.scielo.br/pdf/reben/v60n5/v60n5a19.pdf.

14. Ciconet RM, Marques GQ, Lima MADS. Educação em serviço para profissionais de saúde do Serviço de Atendimento Móvel de Urgência (SAMU): relato de da experiência de Porto Alegre-RS. Interface comunicação saúde educação [periódico na internet]. 2008 [cited 2010 July 09]; 12 (26): 659-66. Available from: http://www.interface. org.br/. 